in 2002-2005 to 2.9 in 2008-2013. This partly reflects an increase in the population of the area covered over the time period (from 750,000 as described in the previous study to 806,683 in the current period).

Comparing earlier and later cohorts, there was a significant increase in the proportion of patients with a specific histological subtype $(36 \%$ vs $86 \%$ respectively, $\mathrm{p}<0.001)$ with a large rise in the proportion of epithelioid cases ( $32 \%$ vs $71 \%$ respectively). Similarly, comparing management of pleural effusion, the use of talc insufflation remained similar ( $38 \%$ vs $44 \%$ respectively) with increased use of indwelling pleural catheters (12\% vs $22 \%$ respectively) and a reduction in talc slurry pleurodesis $(15 \%$ vs $1 \%$ respectively). Overall treatment rates with palliative chemotherapy rose from $18 \%$ to $38 \%(\mathrm{p}=0.0002)$. Median survival rose from 267 days (95\% CI 178-356) to 380 days (95\% CI 252-397) between the two cohorts.

Conclusions The current study shows an unexpected reduction in measured incidence in Leeds, raising the possibility of incomplete case ascertainment in this study period. Specific histological subtyping, rates of palliative chemotherapy, and median survival increased between the cohorts.

\section{P72 INCIDENTAL DETECTION OF EARLY STAGE NON-SMALL CELL LUNG CANCER - TIME TO IMPLEMENT SCREENING?}

${ }^{1} \mathrm{RM}$ Thakrar, ${ }^{1} \mathrm{JM}$ Brown, ${ }^{2} \mathrm{SV}$ Brazil, ${ }^{3} \mathrm{M}$ Nankivell, ${ }^{4} \mathrm{DR}$ Lawrence, ${ }^{5} \mathrm{PJ}$ George, ${ }^{1} \mathrm{SM}$ Janes, ${ }^{5} \mathrm{~N}$ Navani. 'Lungs for Living Research Centre, University College London, UK; ${ }^{2}$ Department of Life Sciences, University College London, UK; ${ }^{3}$ MRC Clinical Trials Unit, London, UK; ${ }^{4}$ The Heart Hospital, London, UK; ${ }^{5}$ University College London Hospital, London, UK

\subsection{6/thoraxjnl-2014-206260.213}

Introduction Early detection is the key to survival in non-small cell lung cancer (NSCLC) where surgical resection can be undertaken. However, stage I and II disease combined account for only $25-30 \%$ of patients presenting with lung cancer. Although, clinical pathways from primary care exist to facilitate expeditious management of patients, the role of other referral pathways to diagnosis of surgically treatable lung cancers is not known.

Methods Patients suitable for surgical resection for curative intent for primary lung cancer were identified between 2007 and 2011 at this institution. Patients diagnosed 'incidentally' on radiology were compared to those detected through the standard

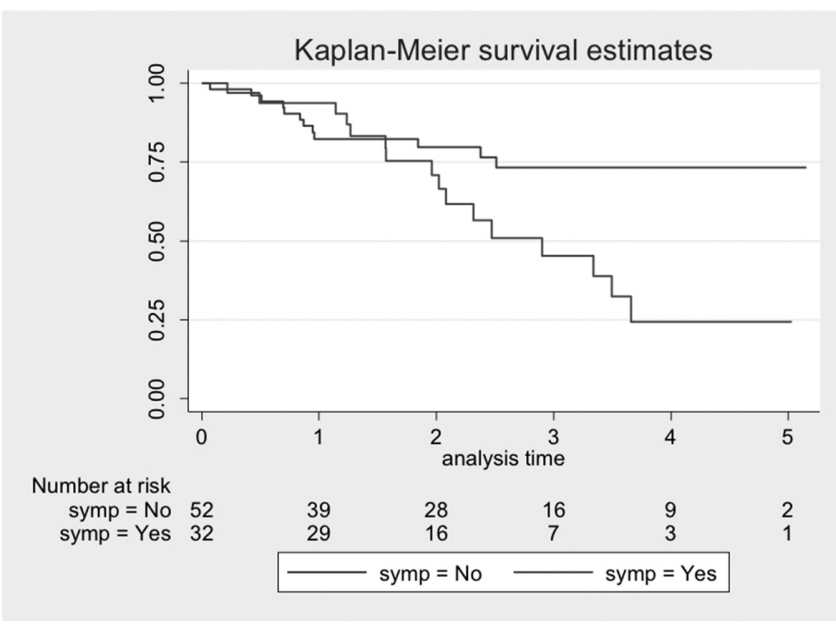

Abstract P72 Figure 1 Kaplan-Meier survival estimates 'two week' wait target referral system. Specific data on demographics, diagnostics utilised, pathological stage, and lung cancer mortality were recorded.

Results Eighty-four patients were treated with surgical resection for non-small cell cancer. The 'two-week' wait referrals accounted for one quarter ( $\mathrm{n}=21 ; 95 \%$ CI $17-35 \%)$ of the all the referrals, whilst $61 \%(\mathrm{n}=51 ; 95 \%$ CI $50-70 \%)$ of patients were found to have lung cancer detected incidentally through investigations performed by other specialties in the hospital. The presentation of patients to hospital with cancer related symptoms whether they had operable disease or not, had significantly higher lung cancer specific mortality ( $p=0.02$; see figure).

Conclusion We demonstrate that patients who have cancerrelated symptoms have a worse outlook. Whilst asymptomatic patients diagnosed by chance have better prospects for cure by surgical resection, thus highlighting the promise of CT screening for lung cancer in patients with high risk factors.

\section{P73 THE RATE OF INCIDENTAL SYNCHRONOUS PATHOLOGY ON PET-CT SCANS PERFORMED FOR THORACIC MALIGNANCY AND SUBSEQUENT IMPACT ON LUNG CANCER PATHWAYS}

TRE Jones, HJ Curtis. Queen Elizabeth Hospital, Gateshead, UK

\subsection{6/thoraxjnl-2014-206260.214}

Introduction NICE (CG121) recommends that all patients potentially suitable for curative intent treatments are offered PET-CT, and that they are treated within 62 days of their urgent referral and within 31 days of the decision to treat.

There are case reports and three large studies regarding incidental findings on PET-CT performed for thoracic malignancy. These studies were based in Sheffield, Australia and Switzerland, with a rate of significant incidental findings of $21 \%, 12 \%$ and $9 \%$ respectively. There are no studies regarding the impact on referral pathways.

We aimed to identify the rate of incidental synchronous pathology on PET-CT for thoracic malignancy in our local population of 190,000 and the impact of these on referral pathways. We serve a local authority district ranked 43/326 in the English Index Multiple Deprivation in 2010 (rank of 1 being most deprived).

Methods Identifying patients from our thoracic MDT database, we retrospectively analysed electronic patient records for those with synchronous pathology on PET-CT between November 2012 and October 2013. Data collected included primary

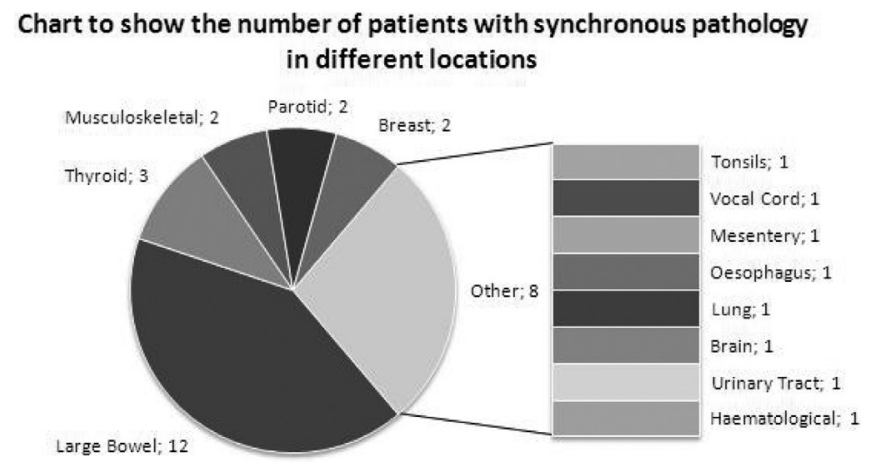

Abstract P73 Figure 1 Chart to show the number of patients with synchronous pathology in different locations 
diagnosis, synchronous pathology, referral timelines and reasons for delays.

Results

- 108 patients had a PET-CT for investigation of thoracic malignancy.

- 29 incidental findings were found in 28 patients (28/ 108;26\%); see chart-1.

- 20 patients $(20 / 108 ; 19 \%)$ required further investigation/referral for their synchronous pathology.

- 22 of these 28 patients were ultimately diagnosed and treated for thoracic malignancy.

- Referral to treatment was delayed in 8 patients $(8 / 108 ; 7 \%)$, but only in $1(1 / 108 ; 1 \%)$ as a direct result of management for their synchronous pathology.

Conclusions A high number of patients undergoing PET-CT have synchronous pathology. Compared with previous UK data we found a higher rate of synchronous pathology. This may reflect a higher burden of disease in the North East of England.

Synchronous findings have a limited impact on referral to treatment pathways. However they can result in more investigations and trips to hospital, which may have a psychological impact on patients already going through a stressful life event. Perhaps this should be highlighted at time of decision to perform PET-CT.

\section{P74 FOLLOW-UP OF LUNG CANCER PATIENTS POST SURGERY}

R Aslam, AR Biswas, P Blaxill. Mid Yorkshire NHS Trust, Wakefield, UK

10.1136/thoraxjn-2014-206260.215

Rationale Over 50\% of patients undergoing surgery for lung cancer die from recurrence or a second episode of lung cancer within 5 yrs. ${ }^{1}$ There is little evidence based guidelines regarding the follow up of post surgical resection. Most follow up with physical examination and plain radiographs. Few recommend follow up with CTs. Within Mid Yorkshire NHS trust, patients are followed up post-operatively for 5 years. In the initial 2 years CTs are performed at 3, 12 and 24 months and chest radiographs at 3 to 6 monthly intervals for 5 years. The aim of this study was to assess the benefits of cross sectional imaging.

Methods A retrospective analysis was conducted of 109 patients undergoing surgery for lung cancer within Mid Yorkshire NHS hospital trust between 2009 and 2012.

Results 109 patients were included in total (42\% female). Types of surgery were lobectomy (80\%), wedge resection (10\%) and pneumonectomy (10\%) Recurrence occurred in 37\% of patients (85\% pulmonary).

$60 \%$ of recurrences were adenocarcinomas and $33 \%$ squamous cell carcinomas. The majority of patients were asymptomatic (78\%). Dyspnoea was the most frequent symptom (19\%). The most commonly staged tumour was $1 \mathrm{~B}$ (pT2A 53\%, pN0 $50 \%)$.

$53 \%$ of recurrences were identified at the 3 month post-operative CT, $8 \%$ at 6 months, $28 \%$ at 12 months and $3 \%$ at 24 months. Chest radiographs identified recurrence at $6(3 \%), 9$ (3\%) $18(3 \%)$ and 21 months (3\%). Total mortality within the recurrence group was $25 \%$.

$54 \%$ of patients had treatment with curative intent (surgery; $23 \%$ radiotherapy; $18 \%$ chemotherapy $8 \%$; chemoradiotherapy $5 \%$. 23\% received palliative treatment, chemotherapy/ radiotherapy.
Conclusions There are substantial benefits of imaging in identifying recurrences in cancer patients. The post-operative CT imaging at 3 and 12 months is advantageous as they identified $53 \%$ and $28 \%$ of the recurrences respectively. However, the benefit of regular chest radiographs and surveillance CT at 24 months is questionable as they were less effective.

\section{REFERENCE}

1 Egerman U, Jaeggi $K$, Habicht JM, Perruchoud AP, Dalugen $P$, Soler M. Regular follow-up after curative resection of nonsmall cell lung cancer: a real benefit for patients? Eur Respir J 2002 Mar;19(3):464-8

\section{P75 PROGNOSTIC IMPLICATIONS OF THE MODIFIED GLASGOW PROGNOSTIC SCORE IN EARLY STAGE NON- SMALL CELL LUNG CANCER}

AM MacKenzie, E Johnson, S Tsim, KG Blyth. Department of Respiratory Medicine, Southern General Hospital, Glasgow, UK

\subsection{6/thoraxjn-2014-206260.216}

Introduction and objectives Up to $50 \%$ of patients treated radically for non-small cell lung cancer (NSCLC) subsequently present with metastatic disease. This is despite rigorous caseselection and the use of adjuvant therapies based on clinical and/ or surgical staging. A simple, objective biomarker that identified patients at higher risk of recurrence might facilitate more effective multi-modality radical treatment.

Since inflammation-based biomarkers offer robust prognostication in metastatic NSCLC, we hypothesised that the modified Glasgow Prognostic Score (mGPS), Neutrophil:Lymphocyte Ratio (NLR) and/or Platelet:Lymphocyte Ratio (PLR), measured prior to radical treatment would have utility in this regard.

Methods Utilising a radiology database, we retrospectively identified all patients with Stage I-IIIa NSCLC who underwent

\section{Survival following Surgical Resection for NSCLC}
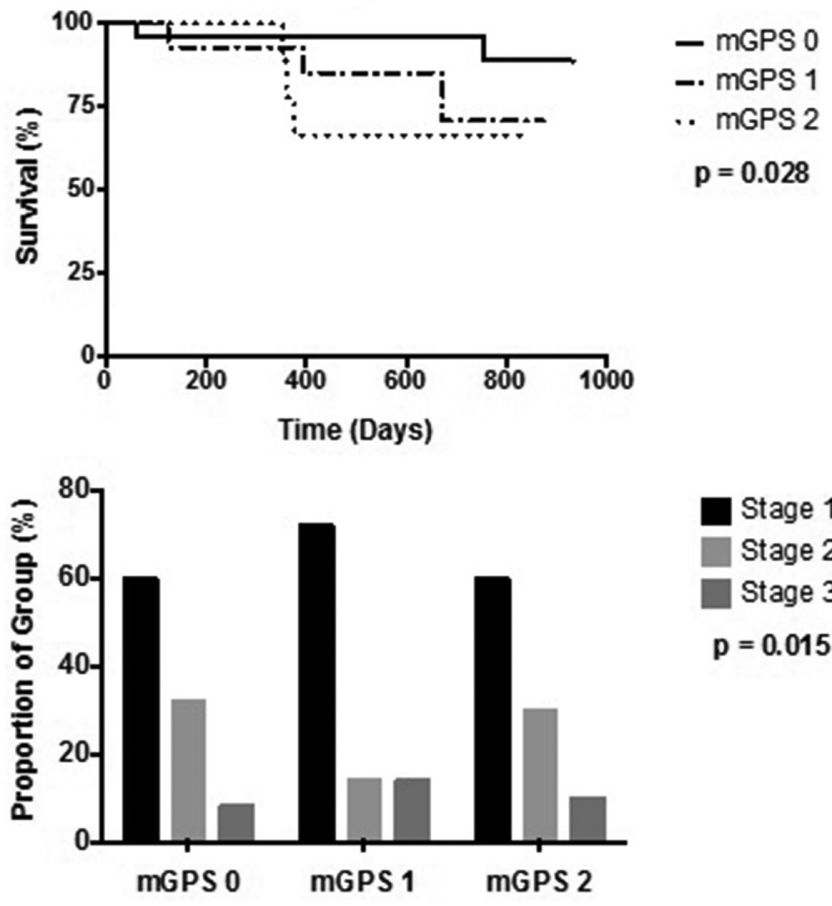

Stage 1

Stage 2

Stage 3

$p=0.015$

Abstract P75 Figure 1 Survival following surgical resection for NSCLC 